\title{
Low mortality of people living with diabetes mellitus diagnosed with COVID-19 and managed at a field hospital in Western Cape Province, South Africa
}

\author{
J-N van der Westhuizen , ${ }^{1} \mathrm{MB}$ ChB; N Hussey, ${ }^{2} \mathrm{MB}$ ChB; M Zietsman, ${ }^{3} \mathrm{MB}$ ChB; N Saldulker, ${ }^{4} \mathrm{MB}$ ChB; K Manning, ${ }^{5} \mathrm{MSc}(\mathrm{Med})$ \\ J A Dave, ${ }^{6}$ FCP, PhD; B Bulajic, ${ }^{7}$ FCEM, MMed (Emerg Med); T Ras, ${ }^{8}$ FCFP, MFamMed \\ ${ }^{1}$ Community Services and Health, City Health, City of Cape Town, South Africa \\ ${ }^{2}$ Department of Medicine, Groote Schuur Hospital, Cape Town, South Africa \\ ${ }^{3}$ Department of Medicine, New Somerset Hospital, Cape Town, South Africa \\ ${ }^{4}$ Metro District Health Services, Western Cape Department of Health, Cape Town, South Africa \\ ${ }^{5}$ Department of Surgery, Faculty of Health Sciences, University of Cape Town and Groote Schuur Hospital, Cape Town, South Africa \\ ${ }^{6}$ Division of Endocrinology, Groote Schuur Hospital and University of Cape Town, South Africa \\ ${ }^{7}$ Division of Emergency Medicine, Faculty of Health Sciences, University of Cape Town, South Africa \\ ${ }^{8}$ Division of Family Medicine, Faculty of Health Sciences, University of Cape Town, South Africa
}

Corresponding author: T Ras (tasleem.ras@uct.ac.za)

\begin{abstract}
Background. The novel coronavirus disease 2019 (COVID-19) was declared an international pandemic by the World Health Organization in March 2020. Throughout the pandemic, the association between diabetes mellitus (DM) and more severe COVID-19 has been well described internationally, with limited data, however, on South Africa (SA). The role of field hospitals in the management of patients with COVID-19 in SA has not yet been described.

Objectives. To describe the mortality and morbidity of people living with DM (PLWD) and comorbid COVID-19, as well as to shed light on the role of intermediate facilities in managing DM and COVID-19 during the pandemic.

Methods. This is a single-centre cross-sectional descriptive study that included all patients with confirmed COVID-19 and pre-existing or newly diagnosed DM (of any type) admitted to the Cape Town International Convention Centre (CTICC) Intermediate Care Bed Facility from June 2020 to August 2020. This study presents the profile of patients admitted to the CTICC, and reports on the clinical outcome of PLWD diagnosed with COVID-19, and additionally determines some associations between risk factors and death or escalation of care in this setting. Results. There were 1447 admissions at the CTICC, with a total of 674 (46.6\%) patients who had confirmed DM, of whom 125 (19\%) were newly diagnosed diabetics and $550(81 \%)$ had pre-existing DM. Included in this group were 57 referrals from the telemedicine platform - a platform that identified high-risk diabetic patients with COVID-19 in the community, and linked them directly to hospital inpatient care. Of the 674 PLWD admitted, 593 were discharged alive, 45 were escalated to tertiary hospital requiring advanced care and 36 died. PLWD who died were older, had more comorbidities (specifically chronic obstructive pulmonary disease, congestive cardiac failure and chronic kidney disease) and were more likely to be on insulin.

Conclusions. In a resource-limited environment, interdisciplinary and interfacility collaboration ensured that complicated patients with DM and COVID-19 were successfully managed in a field hospital setting. Telemedicine offered a unique opportunity to identify highrisk patients in the community and link them to in-hospital monitoring and care. Future studies should explore ways to optimise this collaboration, as well as to explore possibilities for early identification and management of high-risk patients.
\end{abstract}

S Afr Med J 2021;111(10):961-967. https://doi.org/10.7196/SAMJ.2021.v111i10.15779

The novel coronavirus disease 2019 (COVID-19) was declared an international pandemic by the World Health Organization in March 2020. Since then, it has affected and challenged health systems globally, and continues to do so as we are currently confronted with a resurgence of this virus globally. As of 15 March 2021, there were 120504704 confirmed cases, with 2666865 confirmed deaths due to COVID-19. ${ }^{[1]}$

Data on the association between diabetes mellitus (DM) and more severe COVID-19 have been well described internationally. ${ }^{[2]}$ Uncontrolled hyperglycaemia was shown to be an independent risk factor for severe disease and mortality in the last three pandemics of influenza A (H1N1), severe acute respiratory syndrome (SARS) and Middle East respiratory syndrome (MERS) ${ }^{[3-5]}$ - with early evidence also providing extensive links with hyperglycaemia and mortality within the COVID-19 pandemic. ${ }^{[6]}$ Other risk factors, including increasing age, hypertension, cardiovascular disease and obesity have also been associated with more severe COVID-19..$^{[7,8]}$

The prevalence of DM has been increasing globally and South Africa (SA) has not been spared, with some studies estimating a twofold increase in the incidence of type 2 DM from 2000 to 2009 in SA. ${ }^{[9,10]}$ In addition, the International Diabetes Federation suggests that the prevalence of type 2 DM in SA was $12.9 \%$ in 2019, with $52.4 \%$ of people living with diabetes (PLWD) currently undiagnosed. ${ }^{[1]}$

As SA has a relatively young population, it was uncertain what the consequences would be of the colliding pandemics of COVID-19 and DM. Indeed, an early study by Boulle $e t$ al.$^{[12]}$ from the Western Cape Province in SA showed that DM was associated with a 2.4fold increase in mortality when compared with reports from other 
countries. SA already has a healthcare system struggling to cope with the burden of disease related to trauma, communicable (HIV and tuberculosis) and non-communicable diseases, and the extra burden of disease from COVID-19 and the impending morbidity and mortality with associated comorbidities prevalent in the SA population threatened to overwhelm the healthcare services.

Field hospitals have been used for more than a century during epidemics, and provide an important bridge of care when a health system is overwhelmed. ${ }^{[13]}$ The precedent for the use of field hospitals in the COVID-19 pandemic was set by Wuhan City in China, as the city constructed two large field hospitals to help ease the burden on its healthcare system. ${ }^{[14]}$ In line with this, the Western Cape Department of Health commissioned the opening of an 863-bed field hospital to be based at the Cape Town International Convention Centre (CTICC), dubbed the 'Hospital of Hope. The CTICC Intermediate Care Bed Facility (ICBF) served as a field hospital accepting patients with COVID-19 referred from secondary or tertiary level hospitals in the Western Cape.

The role of field hospitals, however, in the management of patients with COVID-19 in SA has not yet been determined. In addition, the clinical outcome of people living with diabetes (PLWD) who were diagnosed with COVID-19 and referred to a field hospital is unknown. Therefore, the aim of this study is to present the profile of patients admitted to the CTICC ICBF and report on the clinical outcome of PLWD diagnosed with COVID-19. Our secondary aims are to determine associations between risk factors and death or escalation of care in this setting.

\section{Methods}

\section{Study design}

This was a single-centre cross-sectional descriptive study, using clinical records as the data source, including all patients with confirmed COVID-19 and pre-existing or newly diagnosed DM (of any type) admitted to the CTICC ICBF from 8 June 2020 to 18 August 2020.

\section{Study site}

The study site was the temporary field hospital for patients with COVID-19 that was established in the building of the CTICC. The large conference hall was adapted to allow for 863 beds allocated into longitudinal wards, equipped with piped oxygen (including the ability to use high-flow nasal oxygen machines), patient bathrooms and pharmacy and nursing stations. The field hospital was staffed with senior managers, junior doctors, physiotherapists, dieticians, social workers and nursing and auxiliary staff, with more than 500 staff employed throughout its open period. Patients meeting the admission criteria were admitted directly from acute hospitals in the Cape Town Metro, and via the community-based telemedicine service. Clinical protocols were drawn from the collective experiences of the surrounding hospitals, and senior specialists from the acute hospitals led weekly clinical rounds.

\section{Population and sampling}

All patients admitted to the facility during the period of operation (8 June 2020 - 18 August 2020) were included in this study. The total sample size was 1447 , of which 674 (46.6\%) patients were identified through records as PLWD (see data collection). All patients admitted to the CTICC ICBF met inclusion and exclusion admission criteria as specified by the facility, and therefore the criteria for admission directly served as criteria for inclusion and exclusion in this study. These criteria included: being $>18$ years of age; having proven
COVID-19; and being demonstrated clinically stable for $>48$ hours in the referring hospital (see appendix A for list of full inclusion and exclusion criteria for admission to CTICC ICBF: http://samj.org.za/ public/sup/15779.pdf)

\section{Telemedicine - a unique sub-cohort}

Early in the COVID-19 pandemic, PLWD were identified as being at high risk for more severe COVID-19. The Western Cape Department of Health launched a project in which PLWD who were deemed to be at very high risk for more severe COVID-19 were identified using databases, and contacted telephonically and offered elective admission to the CTICC ICBF for preventive measures, including diabetes control, oxygen saturation monitoring and symptom monitoring. These patients were identified by a specific task team using databases including the National Health Laboratory Services (NHLS). This was done without physically seeing these patients, and differs from the general cohort in that they were not admitted initially by a referring hospital. The classification of high risk for more severe COVID-19 was ever-evolving alongside new evidence during the pandemic, but initial high-risk criteria were based on PLWD who were $>60$ years of age, with poor diabetes control and/or renal dysfunction with an eGFR $<60 \mathrm{~mL} / \mathrm{min}$ ).

\section{Data collection and analysis}

Data were collected from patient clinical records. Patients with DM were identified (as indicated on the referral, or if newly diagnosed, with an $\mathrm{HbAlc} \geq 6.5 \%$ ) from this larger database of all patients admitted to the CTICC field hospital in Cape Town, SA. The research team developed a data extraction tool that met the objectives of the study, and collected data on demographics (age, gender), medical history (comorbidities and DM management, oxygen requirements, clinical outcome) and laboratory results. The primary outcomes of interest were death, need for escalation of care and discharge home.

Data were entered into a password-protected database hosted by the University of Cape Town. Continuous variables were summarised as medians (interquartile ranges (IQRs)). Categorical variables were expressed as frequencies and percentages. Variables were compared between outcome measures using the Kruskal-Wallis test (nonparametric continuous data), Fisher's exact test (categorical variables with low expected cell frequencies) or $\chi^{2}$ test (categorical variables). Logistic regression was used to assess the associations between clinically relevant outcomes, death and escalation of care. HbAlc was removed from the regression model owing to missing values $(n=156$, $23 \%$ ), and ischaemic heart disease and congestive cardiac failure were excluded from regression owing to the low number of cases. Univariate regression was used to analyse unadjusted associations and retained in the multivariable model to control for confounding. Odds ratios (ORs) were presented with $95 \%$ confidence intervals (CIs). Model diagnostics and Hosmer-Lemeshow goodness-of-fit tests were used to assess model fit. Kaplan-Meier survival curves were presented to demonstrate patient survival and escalation-free survival during CTICC admission.

\section{Ethical considerations}

The study was approved by the Faculty of Health Sciences' Human Research Ethics Committee at the University of Cape Town (ref. no. 502/2020). Approval to access the clinical records was obtained from the Provincial Research Committee of the Western Cape Department of Health. All data were anonymised before being entered into the study dataset, and stored on a password-protected laptop that was only accessible to the research team. 


\section{Results}

There were 1447 admissions to the CTICC, with a total of $674(46.6 \%)$ patients who had confirmed DM, of whom 125 (19\%) were newly diagnosed with DM and 550 (81\%) had pre-existing DM (Fig. 1). Patients with type I DM ( $n=7)$ were included in the analysis.

The demographic baseline characteristics, in-hospital clinical course and outcomes of these patients are described in Table 1. PLWD and those without DM were of similar age and gender, with high levels of comorbidities, such as hypertension (70\%) and chronic kidney disease (CKD) (14\%), both being more common in PLWD. In excess of $80 \%$ of all patients were admitted on supplementary oxygen, and had an equal duration of oxygen requirement (median (IQR) 3 (2 - 6) days). Length of stay (LOS) was slightly longer in PLWD (5 (3 - 8) days), compared with those without DM (4 (2 - 8) days). Outcomes with respect to mortality ( $6 \% \mathrm{v}$. $5 \%$ ), escalation of care $(5 \%$ v. $7 \%)$ and discharge home $(89 \%$ v. 88\%) were similar in PLWD when compared with those without DM.

Of the PLWD, 77\% had received a HbA1c result during the previous 3 months, of whom the majority $(448 / 518,86.5 \%)$ had an HbAlc above the recommended target of 7\% (median (IQR) 10\% (8 - 12\%). Patients with pre-existing DM had a higher HbAlc $(10 \%(8-12 \%))$ compared with those newly diagnosed with DM $(8 \%(7-11 \%))$. In the total group, the majority of patients (64\%) were managed with insulin, with $24 \%$ newly initiated on insulin during their admission.

The telemedicine-recruited cohort $(n=57)$ were older (median age 68 (62 71) years) and had similar baseline clinical characteristics in terms of diabetes control and comorbidities. They differed, however, in their COVID-19 profile from the main cohort in that the majority $(80 \%)$ did not need supplemental oxygen on admission, and had a similar LOS (5 (3 - 7) days), likely due to the clinical protocol applied to them that proposed admission until day 10 of infection.

Of the 674 PLWD admitted, 593 were discharged alive, 45 were escalated to tertiary hospital requiring advanced care and 36 died (Table 2). PLWD who died were older, had more comorbidities (specifically chronic obstructive pulmonary disease, congestive cardiac failure and $\mathrm{CKD}$ ), and were more likely to be on insulin. Although patients discharged and escalated were similar in age, there was a greater proportion of escalated patients who were male, with poor glycaemic control and requiring insulin. Patients who

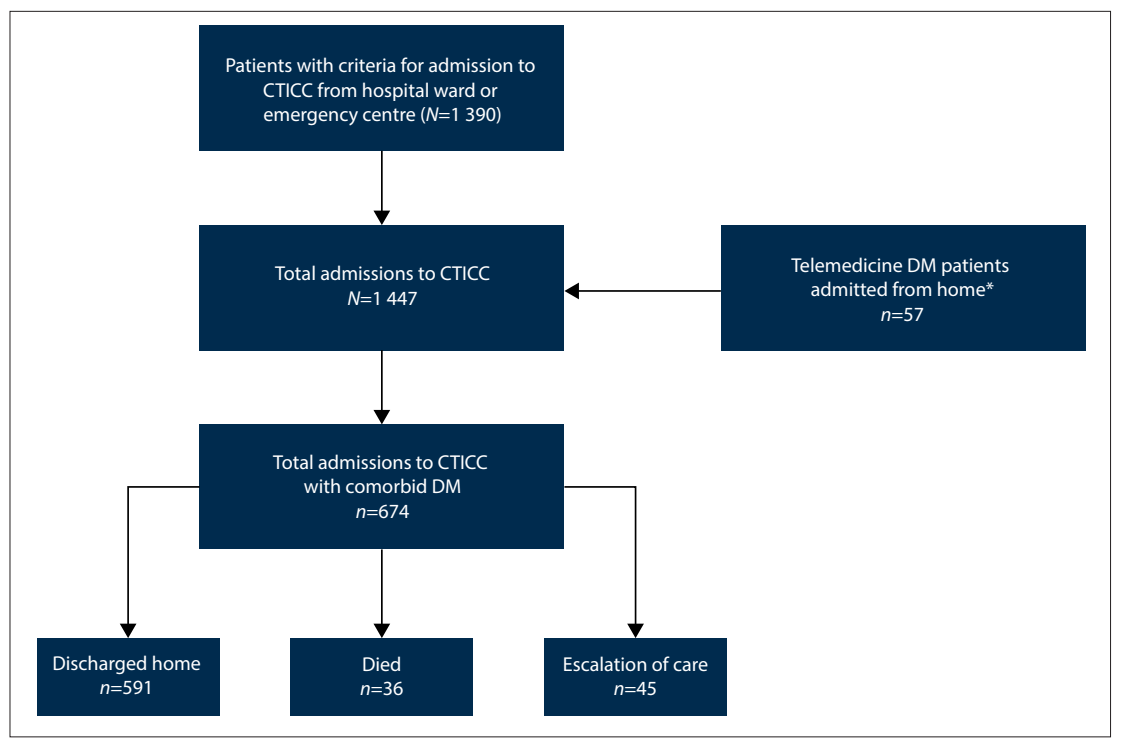

Fig. 1. Flow chart of patients with diabetes mellitus (DM) admitted to the Cape Town International Convention Centre (CTICC) Intermediate Care Bed Facility. For admission criteria see appendix 1 (http://samj.org.za/public/sup/15779.pdf).

${ }^{*}$ Telemedicine diabetic patients with proven COVID-19 and reverse transcription polymerase chain reaction and DM identified via the telemedicine group using information aggregated from the National Health Laboratory Services.

required escalation of care were admitted for a shorter median time of $3(2-7)$ days than those discharged alive $(5(3-8)$ days $)$ and those who died (6 (3 - 11) days).

After multivariate analysis, increasing age (OR 2.19 (95\% CI 1.61 - 3.00) and having CKD (OR 2.50 (95\% CI 1.12 - 5.57) were most significantly associated with increased odds of death (Table 3). Being male and on insulin increased odds of death $>2$, although this was not statistically significant. Being male (OR 2.05 (95\% CI 1.07 - 3.93), on insulin (OR 2.25 (95\% CI $1.04-4.85$ ) and admitted on non-rebreather mask/face-mask oxygen/double-barrel oxygen (OR 7.15, 95\% CI 1.63 - 31.31) were significantly associated with need for escalation of care (Table 4).

Most deaths, escalations of care and discharges occurred within 10 days of admission (Figs 2 and 3). Kaplan-Meier estimates for survival for both death and escalation of care were around $90 \%$ at 10 days post admission.

\section{Discussion}

The present study represents the first report from Africa and the Southern hemisphere on the management of COVID-19 in PLWD at a field hospital. These results show that despite the multiple comorbidities in PLWD and their increased risk of more severe COVID19 , their clinical outcomes in the context of a field hospital were not significantly different to those of people living without diabetes and diagnosed with COVID-19.
A key finding was that almost half (47\%) of all patients admitted to the CTICC were PLWD, despite the estimated population prevalence for DM being 12\%. This finding reinforces the importance of viewing PLWD as a high-risk cohort in the current pandemic.

The patients in our study were more acutely ill than patients in reports from field hospitals elsewhere in the world, as evidenced by the oxygen requirements on admission. By comparison, field hospitals in Wuhan, China, used stricter admission criteria, only admitting patients who were ambulant and had an oxygen saturation of $93 \%$ or more on room air. ${ }^{[14]}$

Of those PLWD who had an HbAlc test result available on admission, only $14 \%$ had an $\mathrm{HbAlc}$ in the recommended target range of $<7 \%$. More concerning was that almost $50 \%$ of PLWD admitted to the CTICC ICBF had an $\mathrm{HbAlc}>10 \%$, confirming the high background levels of poor diabetes control in the community. The high insulin demands were met by using simple insulin regimens and standard COVID-19 treatment protocols, resulting in almost $90 \%$ of PLWD being discharged home. In this cohort, an unexpected finding was that an elevated HbAlC was not predictive of a poor outcome, although our outcomes were influenced by the fact that most patients had been admitted to acute hospitals for a few days before admission to the intermediate care facility. Similarly, Zeming et al. ${ }^{[15]}$ 


\begin{tabular}{|c|c|c|c|}
\hline Total & No DM $(n=771)$ & DM $(n=674)$ & Telemedicine $\mathrm{DM}^{\star}(n=57 / 674)$ \\
\hline Age, median (IQR) & $58(46-69)$ & $60(52-68)$ & $68(62-71)$ \\
\hline \multicolumn{4}{|l|}{ Age category, $n(\%)$} \\
\hline 18 - 39 years & $118(15)$ & $34(5)$ & $0(0)$ \\
\hline $40-59$ years & $300(39)$ & $284(42)$ & $7(12)$ \\
\hline $60-69$ years & $162(21)$ & $215(32)$ & $30(53)$ \\
\hline $70-79$ years & $130(17)$ & $102(15)$ & $17(30)$ \\
\hline$\geq 80$ years & $61(8)$ & $39(6)$ & $3(5)$ \\
\hline \multicolumn{4}{|l|}{ Gender, $n(\%)$} \\
\hline Female & $445(58)$ & $370(55 \%)$ & $28(49)$ \\
\hline Male & $326(42)$ & $304(45 \%)$ & $29(51)$ \\
\hline Newly Dx DM, $n$ (\%) & & $124(18)$ & $0(0)$ \\
\hline \multicolumn{4}{|l|}{ DM type, $n(\%)$} \\
\hline Type I & & $7 / 660(1)$ & $0 / 57(0)$ \\
\hline Type II & & 653/660 (99) & $57 / 57(100)$ \\
\hline \multicolumn{4}{|l|}{ HbAlc category, $n(\%)$} \\
\hline$<7 \%$ & & $70 / 518(14)$ & $8 / 46(17)$ \\
\hline $7-9 \%$ & & 200/518 (39) & $22 / 46(48)$ \\
\hline $10-15 \%$ & & $233 / 518(45)$ & $16 / 46(35)$ \\
\hline$>15 \%$ & & $15 / 518(3)$ & $0 / 46(0)$ \\
\hline Hypertension, $n(\%)$ & $386 / 771(50)$ & $470 / 674(70)$ & $38 / 57(67)$ \\
\hline COPD, $n(\%)$ & 64/771 (8) & $32 / 674(5)$ & $3 / 57(5)$ \\
\hline IHD, $n(\%)$ & $41 / 761(5)$ & $45 / 665(7)$ & $8 / 55(15)$ \\
\hline CCF, $n(\%)$ & $47 / 759(6)$ & $45 / 667(7)$ & $3 / 57(5)$ \\
\hline CKD, $n(\%)$ & $61 / 771(8)$ & $94 / 674(14)$ & $11 / 57(19)$ \\
\hline \multicolumn{4}{|l|}{ Admission $\mathrm{O}_{2}$ category, $n(\%)$} \\
\hline Room air & $97 / 771(13)$ & $118 / 674(18)$ & $46 / 57(81)$ \\
\hline NRB/FM/DB & $232 / 771(30)$ & $215 / 674(32)$ & $1 / 57(2)$ \\
\hline NC & $442 / 771(57)$ & $341 / 674(51)$ & $10 / 57(18)$ \\
\hline Oxygen duration (days), median (IQR) & $3(2-6)$ & $3(2-6)$ & $2(1-4)$ \\
\hline \multicolumn{4}{|l|}{ Patients with DM } \\
\hline On insulin during hospital admission, $n(\%)$ & & $421 / 674(62)$ & $29 / 57(51)$ \\
\hline Chronic insulin, $n(\%)$ & & 255/670 (38) & $21 / 57(37)$ \\
\hline New insulin, $n(\%)$ & & $159 / 670(24)$ & $8 / 57(14)$ \\
\hline No DM medication, $n(\%)$ & & $18 / 670(3)$ & $2 / 57(4)$ \\
\hline Oral medication only, $n$ (\%) & & $238 / 670(36)$ & $26 / 57(46)$ \\
\hline Died, $n(\%)$ & $46(6)$ & $36(5)$ & 2/57 (4) \\
\hline Escalation of care, $n(\%)$ & $36(5)$ & $45(7)$ & $1 / 57(2)$ \\
\hline Discharge home, $n(\%)$ & $689(89)$ & $593(88)$ & $54 / 57(95)$ \\
\hline
\end{tabular}

showed that in 1880 patients managed at a field hospital in Wuhan, China, diabetes did not have an effect on the prognosis of patients. An increasing number of studies are slowly showing that pre-existing poor glycaemic control results in a worse prognosis when diagnosed with COVID-19. Apicella et al. ${ }^{[16]}$ demonstrated that poor glycaemic control before admission was directly related to higher death rates in hospital. In addition, Klonoff et al. ${ }^{[17]}$ showed that admission glucose was a strong predictor of death among patients directly admitted to the intensive care unit (ICU), and that a blood glucose level $>13.88 \mathrm{mmol} / \mathrm{L}$ soon after admission (days 2 - 3) was a strong predictor of death among non-ICU patients. The relatively good outcomes experienced in the present study may be a reflection of the good interfacility engagements seen in Cape Town during the first wave of the pandemic in 2020, and may be appropriate for a field hospital, where complex and severely ill patients are retained within the acute care environment. This points to the desirability of a systematic, collaborative and inclusive pandemic response that cuts across all levels of care.

Patients who required an escalation of their care were more likely to be younger (owing to having acceptable criteria for ICU), be male, have high oxygen demands and require insulin. They were admitted for a significantly shorter duration than the median, and therefore the assumption can be made that they were prematurely referred from referral hospitals. Further studies will have to compare these 
Table 2. Comparison of demographic and clinical characteristics by outcome in PLWD ( $N=674)$

\begin{tabular}{|c|c|c|c|c|}
\hline Characteristic & Discharge home $(n=593)$ & Escalation of care $(n=45)$ & Died $(n=36)$ & $p$-value \\
\hline Age, median (IQR) & $60(52-68)$ & $60(52-66)$ & $70(60-80)$ & $<0.001$ \\
\hline \multicolumn{5}{|l|}{ Age category, years, $n(\%)$} \\
\hline $18-39$ & $32(5)$ & $2(4)$ & $0(0)$ & \multirow[t]{5}{*}{$<0.001$} \\
\hline $40-59$ & $256(43)$ & $20(44)$ & $8(22)$ & \\
\hline $60-69$ & $187(32)$ & $18(40)$ & $10(28)$ & \\
\hline $70-79$ & $89(15)$ & $4(9)$ & $9(25)$ & \\
\hline$\geq 80$ & $29(5)$ & $1(2)$ & $9(25)$ & \\
\hline \multicolumn{5}{|l|}{ Gender, $n(\%)$} \\
\hline Female & $338(57)$ & $17(38)$ & $15(42)$ & \multirow[t]{2}{*}{0.012} \\
\hline Male & $255(43)$ & $28(62)$ & $21(58)$ & \\
\hline Duration of symptoms, days, median (IQR) & $7(4-7)$ & $7(3-9)$ & $7(3-7)$ & 0.220 \\
\hline Newly Dx DM, $n(\%)$ & $112(19)$ & $9(20)$ & $3(8)$ & 0.280 \\
\hline \multicolumn{5}{|l|}{ DM type, $n(\%)$} \\
\hline Type I & $6 / 580^{*}(1)$ & $1 / 44^{*}(2)$ & $0 / 36(0)$ & \multirow[t]{2}{*}{0.600} \\
\hline Type II & $574 / 580^{*}(99)$ & $43 / 44^{*}(98)$ & $36 / 36(100)$ & \\
\hline HbAlc, \%, median (IQR) & $10(8-12)$ & $11(8-13)$ & $9(7-10)$ & 0.110 \\
\hline Hypertension, $n(\%)$ & $408 / 593(69)$ & $33 / 45(73)$ & $29 / 36(81)$ & 0.280 \\
\hline COPD, $n(\%)$ & $22 / 593(4)$ & $5 / 45(11)$ & $5 / 36(14)$ & 0.002 \\
\hline IHD, $n(\%)$ & $39 / 584(7)$ & $5 / 45(11)$ & $1 / 36(3)$ & 0.360 \\
\hline CCF, $n(\%)$ & $34 / 586(6)$ & $5 / 45(11)$ & $6 / 36(17)$ & 0.020 \\
\hline $\mathrm{CKD}, n(\%)$ & $70 / 593(12)$ & $9 / 45(20)$ & $15 / 36(42)$ & $<0.001$ \\
\hline Admission on $\mathrm{O}_{2}$ category, $n(\%)$ & $485 / 593(82)$ & $43 / 45(95)$ & $28 / 36(78)$ & $<0.001$ \\
\hline Oxygen duration, days, median (IQR) & $3(2-5)$ & $3(2-6)$ & $7(3-9)$ & 0.130 \\
\hline \multicolumn{5}{|l|}{ Diabetes medication, $n$ (\%) } \\
\hline On insulin (in hospital), $n$ (\%) & $359(61)$ & $36(80)$ & $26(72)$ & 0.016 \\
\hline Required steroid Rx, $n(\%)$ & $342(58)$ & $35(78)$ & $25(69)$ & 0.014 \\
\hline AKI, $n(\%)$ & $94(16)$ & $11(24)$ & $9(25)$ & 0.140 \\
\hline Hypoglycaemia during admission, $n(\%)$ & $86 / 581(15)$ & $8 / 43(19)$ & $7(19)$ & 0.620 \\
\hline Length of stay, days, median (IQR) & $5(3-8)$ & $3(2-7)$ & $6(3-11)$ & 0.020 \\
\hline
\end{tabular}

Table 3. Associations between risk factors and death in PLWD

\begin{tabular}{|c|c|c|c|c|}
\hline Risk factor & Univariable OR (95\% CI) & $p$-value & Multivariable OR (95\% CI) & $p$-value \\
\hline Age $^{*}$ & $2.19(1.61-3.00)$ & $<0.001$ & $2.12(1.50-2.99)$ & $<0.001$ \\
\hline Male sex & $1.85(0.94-3.66)$ & 0.077 & $2.09(0.98-4.42)$ & 0.055 \\
\hline On insulin & $1.68(0.80-3.56)$ & 0.171 & $2.01(0.89-4.57)$ & 0.095 \\
\hline CKD & $5.31(2.61-10.8)$ & $<0.001$ & $2.50(1.12-5.57)$ & 0.025 \\
\hline Hypertension & $1.89(0.81-4.40)$ & 0.138 & $1.34(0.54-3.32)$ & 0.533 \\
\hline \multicolumn{5}{|c|}{ On $\mathrm{O}_{2}$ at admission (ref. RA) } \\
\hline $\mathrm{NRB} / \mathrm{FM} / \mathrm{DB}$ & $1.38(0.58-3.28)$ & 0.468 & $1.93(0.76-4.95)$ & 0.169 \\
\hline $\mathrm{NC}$ & $0.43(0.16-1.10)$ & 0.080 & $0.53(0.19-1.47)$ & 0.223 \\
\hline
\end{tabular}

patients' outcomes against those who stayed at tertiary hospitals, as the transfer and delay of emergency care potentially has a big impact.

Our findings on increased risk of death in older patients and males is consistent with descriptive results reported in a national epidemiological study by Pillay-van Wyk et al. ${ }^{[18]}$ Older patients are more likely to have CKD and comorbidities, which likely contribute to additional risk. The higher death rate in males was also reported in other studies. ${ }^{[19]}$ Yang et al. ${ }^{[20]}$ found odds of death in males to be
3.8 (95\% CI 1.57 - 9.50) compared with females. This has implications for the rapid identification of patients who are most at risk of death.

The low mortality associated with those patients identified by the telemedicine services using the aforementioned risk factors as key referral criteria suggests that when these high-risk patients are identified early, and proactively admitted into a controlled environment, their clinical outcomes can be positively affected. The telemedicine service and the specific outcomes need further research 


\begin{tabular}{|c|c|c|c|c|}
\hline Risk factor & Univariable OR (95\% CI) & $p$-value & Multivariable OR $(95 \% \mathrm{CI})$ & $p$-value \\
\hline Age $^{*}$ & $0.96(0.74-1.23)$ & 0.721 & $1.00(0.75-1.32)$ & 0.995 \\
\hline Male sex & $2.18(1.17-4.07)$ & 0.014 & $2.05(1.07-3.93)$ & 0.030 \\
\hline On insulin & $2.59(1.23-5.48)$ & 0.013 & $2.25(1.04-4.85)$ & 0.038 \\
\hline CKD & $1.86(0.86-4.02)$ & 0.116 & $1.69(0.73-3.91)$ & 0.220 \\
\hline Hypertension & $1.26(0.63-2.49)$ & 0.513 & $1.24(0.61-2.54)$ & 0.545 \\
\hline \multicolumn{5}{|c|}{ On $\mathrm{O}_{2}$ at admission (ref. RA) } \\
\hline NRB/FM/DB & $7.35(1.70-31.74)$ & 0.007 & $7.15(1.64-31.31)$ & 0.009 \\
\hline NC & $3.23(0.74-14.13)$ & 0.118 & $2.94(0.66-13.01)$ & 0.155 \\
\hline
\end{tabular}

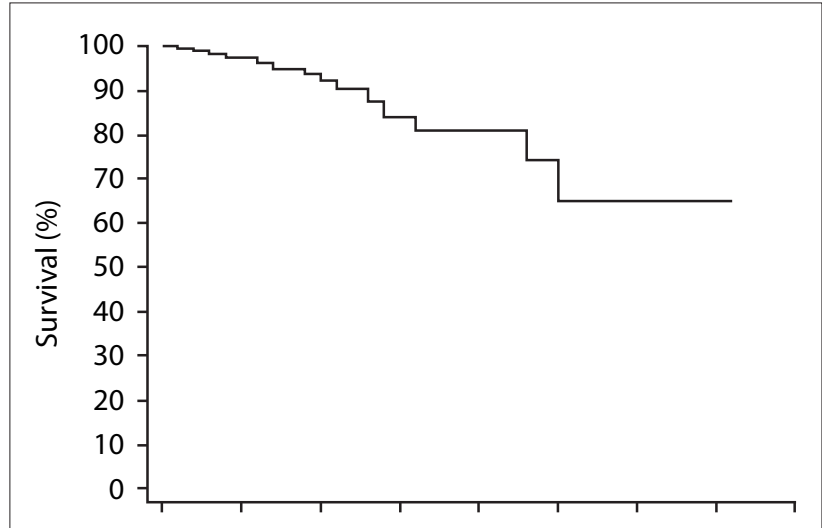

Days since CTICC admission, $n$

Fig. 2. Kaplan-Meyer curve showing time to death for patients with COVID-19 and diabetes mellitus. $($ CTICC $=$ Cape Town International Convention Centre.)

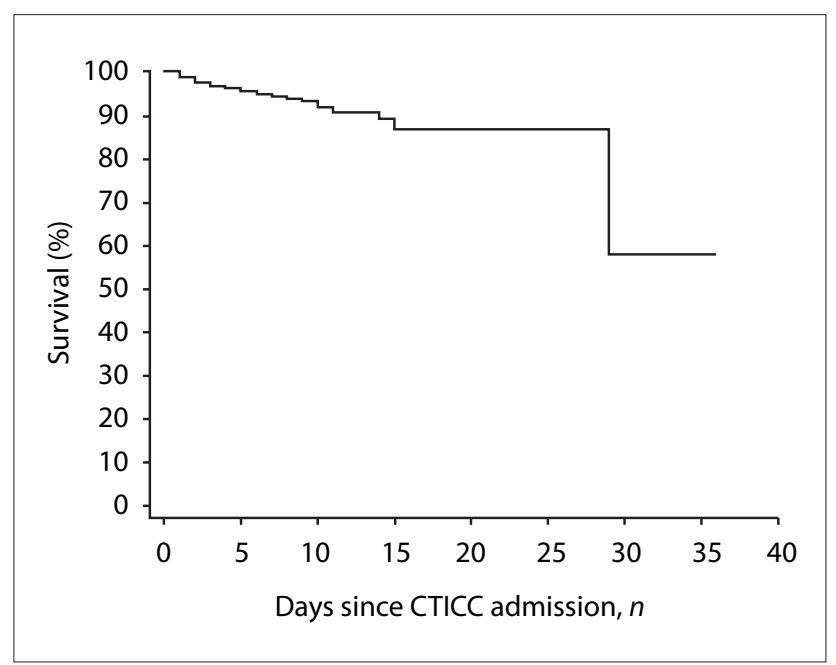

Fig. 3. Kaplan-Meyer curve showing time to escalation of care for patients with COVID-19 and diabetes mellitus. (CTICC = Cape Town International Convention Centre.)

to understand the role of preventive medicine during a pandemic, with consideration and investigation of the cost-benefit ratio and the added burden it places on already overwhelmed health services. It served as an interesting pilot study for integrating existing technology (e.g. the NHLS databases) and telephonic communications in a proactive response to a developing pandemic. It also serves as a possible area of innovation for future facilities to integrate these services into their package of care.

There were several limitations to this study. Key clinical variables such as daily serum glucose levels and body mass index were not included in the clinical dataset. This meant that we were not able to comment on the impact of in-patient glycaemic control or obesity on the clinical outcomes. The data are only descriptive of an intermediate care facility, and the outcomes should be interpreted in this context.

No records regarding outcome were kept for the 45 patients who required escalation of care, of whom 44 were PLWD, and therefore we are unable to report on the outcome of this subset of patients admitted to the CTICC ICBF. Follow-up of the outcomes of this group of patients would be of value to comment on the overall outcome of PLWD admitted to CTICC ICBF. However, it remains reassuring and significant that at least $88 \%$ of PLWD, who represent one of the highest-risk groups for more severe COVID-19, were discharged alive from the CTICC ICBF.

The strengths of this study rest with it being the first description of the use of a field hospital in SA to manage high-risk patients diagnosed with COVID-19. With resource limitations and lack of access to hospital beds being rife in certain areas of SA and in other low- and middle-income countries, this study provides important data for a cost-effective model to show its success in managing highrisk patients during a pandemic.

\section{Conclusion}

In a resource-limited environment, interdisciplinary and interfacility collaboration ensured that PLWD and COVID-19 were successfully managed in a field hospital setting. Future studies should explore ways to optimise this collaboration, as well as to explore possibilities for early identification and management of high-risk patients.

Declaration. None.

Acknowledgements. None.

Author contributions. Equal contributions.

Funding. None.

Conflicts of interest. None.

1. Worldometer. COVID 19 - coronavirus disease pandemic. https://www.worldometers.info/ coronavirus/ (accessed 15 March 2021)

2. Palaiodimos L, Chamorro-Pareja N, Karamanis D, et al. Diabetes is associated with increased risk for in-hospital mortality in patients with COVID-19: A systematic review and meta-analysis comprising 18506 patients. Hormones 2021;20(2):305-314. https://doi.org/10.1007/s42000-020-00246-2

Y Yang JK, Feng Y, Yuan MY, et al. Plasma glucose levels and diabetes are independent predictors for mortality and morbidity in patients with SARS. Diabet Med 2006;23(6):623-628. https://doi. org/10.1111/j.1464-5491.2006.01861.x

4. Song Z, Xu Y, Bao L, et al. From SARS to MERS, thrusting coronaviruses into the spotlight. Viruses 2019;11(1):59. https://doi.org/10.3390/v11010059 
5. Schoen K, Horvat N, Guerreiro NFC, de Castro I, de Gi- assi KS. Spectrum of clinical and radiographic findings in patients with diagnosis of H1N1 and correlation with clinical severity. BMC Infect Dis findings in patients with diagnosis of H1N1 and corre
2019;19:964. https://doi.org/10.1186/s12879-019-4592-0

6. Carrasco-Sánchez FJ, López-Carmona MD, Martínez-Marcos FJ, et al. Admission hyperglycemia as a predictor of mortality in patients hospitalized with COVID-19 regardless of diabetes status: Data a predictor of mortality in patients hospitalized with COVID-19 regardless of diabetes status: Data
from the Spanish SEMI-COVID-19 Registry. Ann Med 2021:53(1):103-116. https://doi.org/10.1080/ from the Spanish SEM

7. Suleyman G, Fadel RA, Malette KM, et al. Clinical characteristics and morbidity associated with coronavirus disease 2019 in a series of patients in metropolitan Detroit. JAMA Netw Open 2020;3(6):e2012270. https://doi.org/10.1001/jamanetworkopen.2020.12270

8. Barry M, Althabit N, Akkielah L, et al. Clinical characteristics and outcomes of hospitalized COVID-19 patients in a MERS-CoV referral hospital during the peak of the pandemic. Int J Infect Dis 2021;106(43-51). https://doi.org/10.1016/j.ijid.2021.03.058

9. Bradshaw D, Norman R, Pieterse D, et al. Estimating the burden of disease attributable to diabetes in South Africa in 2000. S Afr Med J 2007;97:700-706.

10. Bertram MY, Jaswal AV, Van Wyk VP, Levitt NS, Hofman KJ. The non-fatal disease burden caused by type 2 diabetes in South Africa, 2009. Glob Health Action 2013;24(6):19244. https://doi.org/10.3402/ gha.v6i0.19244

11. International Diabetes Federation. IDF Diabetes Atlas. 9th edition. Brussels: IDF, 2019. https://www. diabetesatlas.org/upload/resources/2019/IDF_Atlas_9th_Edition_2019.pdf (accessed 30 March 2021).

12. Boulle A, Davies M, Hussey H, et al. Risk factors for COVID-19 death in a population cohort study from the Western Cape Province, South Africa. Clin Infect Dis 2020: ciaal198. https://doi. org/10.1093/cid/ciaal198

13. Luo H, Liu J, Li C, Chen K, Zhang M. Ultra-rapid delivery of specialty field hospitals to combat COVID-19: Lessons learned from the Leishenshan Hospital project in Wuhan. Autom Constr 2020;119:103345. https://doi.org/10.1016/jautcon.2020.103345

14. Chen S, Zhang Z, Yang J, et al. Fangcang shelter hospitals: A novel concept for responding to public health emergencies. Lancet 2020;395(10232):1305-1314. https://doi.org/10.1016/S0140 6736(20)30744-3
15. Zeming Liu, Jinpeng Li, Jianglong Huang, et al. Association between diabetes and COVID-19: A retrospective observational study with a large sample of 1880 cases in Leishenshan Hospital, Wuhan Front Endocrinol 2020;11:478. https://doi.org/10.3389/fendo.2020.00478

16. Apicella M, Campopiano MC, Mantuano M, Mazoni L, Coppelli A, Del Prato S. COVID-19 in people with diabetes: Understanding the reasons for worse outcomes. Lancet Diab Endocrinol 2020;8(9):782792 (erratum in: Lancet Diabetes Endocrinol 2020;8(10):e5; erratum in: Lancet Diabetes Endocrinol 792 (erratum in: Lancet Diabetes Endocrinol 2020;8(10):e5; er
2020;8(11):e6). https:///oi.org/10.1016/S2213-8587(20)30238-2

17. Klonoff DC, Messler JC, Guillermo E, et al. Association between achieving inpatient glycemic contro and clinical outcomes in hospitalized patients with COVID-19: A multicenter, retrospective hospitalbased analysis. Diabetes Care 2021;44(2):578-585. https://doi.org/10.2337/dc20-1857

18. Pillay-Van Wyk V, Bradshaw D, Groenewald P, et al. COVID-19 deaths in South Africa: 99 days since South Africa’s first death. S Afr Med J 2020;110(11):1093-1099. https://doi.org/10.7196/SAMJ.2020. v110i11.15249

19. Ng J, Bakrania K, Russell R, Falkous C. COVID-19 mortality rates by age and gender: Why is the disease killing more men than women? RGA, 3 July 2020. https://www.rgare.com/docs/default-source/ knowledge-center-articles/covid-19-case-fatality agegender.pdf?sfursn=5806a7ea 2 (accessed 29 January 2021).

20. Yang $\mathrm{K}$, Sheng $\mathrm{Y}$, Huang $\mathrm{C}$, et al. Clinical characteristics, outcomes, and risk factors for mortality in patients with cancer and COVID-19 in Hubei, China: A multicentre, retrospective, cohort study in patients with cancer and COVID-19 in Hubei, China: A multicentre, retrospective

21. Western Cape Government. Covid-19 intermediate care bed facilities clinical care plan. Version 3 (final). Cape Town: Western Cape Government, 2020.

Accepted 9 June 2021. 